\title{
PROXIMITY RELATIONS IN TRANSFORMATION GROUPS
}

\author{
BY \\ JESSE PAUL CLAY(1)
}

Introduction. Let $(X, T)$ be a transformation group whose phase space $X$ is a uniform space. Most of the results are stated for compact $X$. In this paper, four proximity relations, $L, M, P$, and $Q$, in $X$ are defined and some of their properties studied. The relations $P$ and $Q$ were first defined and studied by Ellis and Gottschalk [5]. As a general reference for the notions occurring here, consult $[8]$.

In Theorem $1, L$ is shown to be an invariant equivalence relation in $X$, and in Theorem 3, $L$ is characterized as the union of all orbit closures under $(X \times X, T)$ which are contained in $P$. Theorem 3 also shows that if $P$ is closed, then $P=L$ and $P$ is a closed invariant equivalence relation in $X$. Theorem 4 establishes the productivity of the relations $L$ and $M$, and in Theorem 7 , it is shown that $(X, T)$ is coterminous, i.e., $P=Q=L=M$, iff $P=Q$. Theorems 9 through 13 describe $L, M, P$, and $Q$ under various hypotheses such as $(X, T)$ distal; $(X, T)$ minimal and $T$ abelian; $(X, T)$ regionally mixing; and the like.

Theorem 14 is an application of the general theory to obtain a characterization of $(X, T)$ being uniformly equicontinuous, and as such represents a strengthening of a theorem by John D. Baum [2].

The author is indebted to Professor W. H. Gottschalk for his invaluable suggestions, mathematical stimulation, and sustained interest.

StANDING NOTATION. Let $(X, T)$ be a transformation group where $X$ is always a uniform space. Let $\mathscr{A}$ be the class of all syndetic subsets of $T$, let $\mathscr{K}$ be the class or all compact subsets of $T$, let $\mathscr{U}$ be the uniformity of $X$, and for each $x \in X$ let $\mathscr{N}_{x}$ be the neighborhood filter of $x$.

Definition 1. (1) The (simply) proximal relation of $(X, T)$ denoted $P(X, T)$ of $P(X)$ or $P_{X}$ or $P$, is defined to be the set of all $(x, y) \in X \times X$ such that if $\alpha \in \mathscr{U}$, then there exists $t \in T$ such that $(x t, y t) \in \alpha$.

(2) The regionally (simply) proximal relation of $(X, T)$, denoted $Q(X, T)$ or $Q(X)$ or $Q_{X}$ or $Q$, is defined to be the set of all $(x, y) \in X \times X$ such that if

Received by the editors June 21, 1962.

(1) This research was partially supported by the United States Air Force through the Air Force Office of Scientific Research of the Air Research and Development Command, under Contract No. AF49(638)-569. Reproduction in whole or in part is permitted for any purpose of the United States Government. 
$\alpha \in \mathscr{U}$, if $U \in \mathscr{N}_{x}$, and if $V \in \mathscr{N}_{y}$, then there exist $x_{1} \in U, y_{1} \in V$, and $t \in T$ such that $\left(x_{1} t, y_{1} t\right) \in \alpha$.

(3) The syndetically proximal relation of $(X, T)$, denoted $L(X, T)$ or $L(X)$ or $L_{X}$ or $L$, is defined to be the set of all $(x, y) \in X \times X$ such that if $\alpha \in \mathscr{U}$, then there exists $A \in \mathscr{A}$ such that $t \in A$ implies $(x t, y t) \in \alpha$.

(4) The regionally syndetically proximal relation of $(X, T)$, denoted $M(X, T)$ or $M(X)$ or $M_{X}$ or $M$, is defined to be the set of all $(x, y) \in X \times X$ such that if $\alpha \in \mathscr{U}$, if $U \in \mathscr{N}_{x}$, and if $V \in \mathscr{N}_{y}$, then there exist $x_{1} \in U, y_{1} \in V$, and $A \in \mathscr{A}$ such that $\left(x_{1}, y_{1}\right) A \subset \alpha$.

REMARK 1. The following statements hold:

(1) $L=\bigcap_{\alpha \in \mathscr{U}} \bigcup_{K \in \mathscr{K}} \bigcap_{t \in T} \alpha K t$,

(2) $M=\bigcap_{\alpha \in \mathscr{U}} \overline{\bigcup_{K \in \mathscr{X}} \bigcap_{t \in T} \alpha K t}$,

(3) $P=\bigcap_{\alpha \in \mathscr{U}} \alpha T$,

(4) $Q=\bigcap_{\alpha \in \mathscr{U}} \overline{\alpha T}$,

(5) $\Delta \subset L \subset M \subset Q \subset X \times X$ and $\Delta \subset L \subset P \subset Q \subset X \times X$. Here $\Delta=\{(x, x) \mid x \in X\}$.

(6) $L, M, P$, and $Q$ are invariant reflexive symmetric relations in $X$. $M$ and $Q$ are closed in $X \times X$.

Definition AND REMARK 2. The transformation group $(X, T)$ is said to be repletely distal provided that the following equivalent statements are satisfied:

(1) $L=\Delta$.

(2) If $x, y \in X$ with $x \neq y$, then there exists $\alpha \in \mathscr{U}$ and a replete subset $A$ of $T$ such that $(x, y) A \subset \alpha^{\prime}$.

Definition AND REMARK 3. The transformation group $(X, T)$ is said to be regionally repletely distal provided that the following equivalent statements are satisfied:

(1) $M=\Delta$.

(2) If $x, y \in X$ with $x \neq y$, then there exist $\alpha \in \mathscr{U}, U \in \mathscr{N}_{x}$, and $V \in \mathscr{N}_{y}$ such that if $x_{1} \in U$ and if $y_{1} \in V$, then there exists a replete subset $A$ of $T$ such that $\left(x_{1}, y_{1}\right) A \subset \alpha^{\prime}$.

STANDING HYPOTHESIS. Henceforth we will assume that all transformation groups have phase spaces which are compact separated uniform spaces unless statements to the contrary are specifically made.

The purpose of the next three theorems (and associated remarks) is to point out some general facts about the basic structure of the relations $P$ and $L$.

LeMma 1. Let $T$ be a group, and let $A_{1}, A_{2}, K_{1}, K_{2}, B$ be subsets of $T$ such that $A_{1} K_{1} \supset A_{2}$ and $A_{2} K_{2} \supset B$. Then $\left(A_{1} \cap A_{2} K_{1}^{-1}\right) K_{1} K_{2} \supset B$. 
Proof. Let $b \in B$. There exist $a_{2} \in A_{2}$ and $k_{2} \in K_{2}$ such that $b=a_{2} k_{2}$. Choose $a_{1} \in A_{1}$ and $k_{1} \in K_{1}$ for which $a_{2}=a_{1} k_{1}$. Now $b=a_{1} k_{1} k_{2}$ and $a_{1}=a_{2} k_{1}^{-1} \in A_{1} \cap A_{2} K_{1}^{-1}$. The proof is completed.

LEMmA 2. Let $T$ be a group, and let $A_{1}, A_{2}, K_{1}, K_{2}$ be subsets of $T$ such that $T=A_{1} K_{1}=A_{2} K_{2}$. Then $T=\left(A_{1} \cap A_{2} K_{1}^{-1}\right) K_{1} K_{2}$.

Proof. Use Lemma 1.

LEMMA 3. Let $T$ be a group, let $n$ be a positive integer, and let $A_{1}, \cdots, A_{n}, K_{1}, \cdots, K_{n}$ be subsets of $T$ such that $T=A_{i} K_{i}$ for $i=1, \cdots, n$ and let $K_{0}$ be the identity element of $T$. Then

$$
T=\left(\bigcap_{i=1}^{n} A_{i}\left(\prod_{j=1}^{i-1} K_{j}\right)^{-1}\right) \prod_{i=1}^{n} K_{i}
$$

Proof. We prove the lemma by induction. The statement is clearly true for $n=1$. Let $p$ be a positive integer and assume the statement is true for $n=p$. We show the statement is true for $n=p+1$. By hypothesis,

$$
T=\left(\bigcap_{i=1}^{p} A_{i}\left(\prod_{j=1}^{i-1} K_{j}\right)^{-1}\right) \prod_{i=1}^{p} K_{i} \text { and } T=A_{p+1} K_{p+1}
$$

From Lemma 2, it follows that

$$
\begin{aligned}
T & =\left(\left(\bigcap_{i=1}^{p} A_{i}\left(\prod_{j=1}^{i-1} K_{j}\right)^{-1}\right) \cap A_{p+1}\left(\prod_{i=1}^{p} K_{i}\right)^{-1}\right)\left(\prod_{i=1}^{p} K_{i}\right) K_{p+1} \\
& =\left(\bigcap_{i=1}^{p+1} A_{i}\left(\prod_{j=1}^{i-1} K_{j}\right)^{-1}\right) \prod_{i=1}^{p+1} K_{i} .
\end{aligned}
$$

The proof is completed.

THEOREM 1. Let $(X, T)$ be a transformation group. Then $L$ is an invariant equivalence relation in $X$.

Proof. It remains only to show that $L$ is transitive in $X$. Let $(x, y) \in L$ and $(y, z) \in L$. We show $(x, z) \in L$. Let $\alpha \in \mathscr{U}$. Choose $\beta \in \mathscr{U}$ such that $\beta^{2} \subset \alpha$. There exist $A_{1} \in \mathscr{A}$ and $K \in \mathscr{K}$ such that $T=A_{1} K$ and $(x, y) A_{1} \subset \beta$. There exists $A_{2} \in \mathscr{A}$ such that $(y, z) A_{2} K^{-1} \subset \beta$. Set $A=A_{1} \cap A_{2} K^{-1}$. Now $(x, y) A \subset \beta$ and $(y, z) A \subset \beta$, whence $(x, z) A \subset \alpha$. Since $A$ is syndetic by Lemma $2,(x, z) \in L$. The proof is completed.

THEOREM 2. Let $(X, T)$ be a transformation group. Then $L P \cup P L=P$.

Proof. It is sufficient to show $P L \subset P$ and $L P \subset P$. Let $(x, z) \in P L$. We show $(x, z) \in P$. Let $\alpha \in \mathscr{U}$ and $y \in X$ such that $(x, y) \in P$ and $(y, z) \in L$. Choose $\beta \in \mathscr{U}$ such that $\beta^{2} \subset \alpha$. There exist $A \in \mathscr{A}, t \in T$, and $K \in \mathscr{K}_{i}$ such that $(y, z) A \subset \beta$ and 
$(x, y) t K^{-1} \subset \beta$ and $T=A K$. Choose $s \in t K^{-1} \cap A$. Now $(x, z) s \in \beta^{2} \subset \alpha$, whence $(x, z) \in P$. Similarly, $L P \subset P$. The proof is completed.

Lemma 4. Let $(X, T)$ be a transformation group where $X$ is not necessarily compact, and let $z \in L$. Then $\overline{z T} \subset L$.

Proof. Let $w \in \overline{z T}$. We show $w \in L$. Let $\alpha \in \mathscr{U}$ with $\alpha=\bar{\alpha}$. There exists $A \in \mathscr{A}$ such that $z A \subset \alpha$. Let $K \in \mathscr{K}$ such that $T=A K$. It is enough to show $w t K^{-1} \cap \alpha \neq \varnothing$ for all $t \in T$. Let $t \in T$. Assume $w t K^{-1} \cap \alpha=\varnothing$, that is, $w t K^{-1} \subset \alpha^{\prime}$. There exists $U \in \mathscr{N}_{w}$ such that $U t K^{-1} \subset \alpha^{\prime}$, that is $U t K^{-1} \cap \alpha=\varnothing$. Choose $s \in T$ such that $z s \in U$. Since $s t \in T=A K$, it follows that $s t K^{-1} \cap A \neq \varnothing$, $z s t K^{-1} \cap z A \neq \varnothing$ and $z A \notin \alpha$. This is a contradiction. The proof is completed.

Lemma 5. Let $(X, T)$ be a transformation group, and let $z \in X \times X$ such that $\overline{z T} \subset P$. Then $z \in L$.

Proof. Let $\alpha$ be an open index of $X$. For each $w \in \overline{z T}$, there exists $t_{w} \in T$ such that $w t_{w} \in \alpha$ and hence there exists an open neighborhood $U_{w}$ of $w$ in $X \times X$ such that $U_{w} t_{w} \subset \alpha$. Choose a finite subset $F$ of $\overline{z T}$ so that $\overline{z T} \subset \bigcup_{w \in F} U_{w}$. Define $K=\left\{t_{w} \mid w \in F\right\}$ and $A=\{t \mid t \in T . z t \in \alpha\}$. It is enough to show that $T=A K^{-1}$. Let $t \in T$. Then $z t \in \overline{z T} \subset \bigcup_{w \in F} U_{w}$ and there exists $f \in F$ such that $z t \in U_{f}$ whence $z t t_{f} \in \alpha$ and $t \in A K^{-1}$. The proof is completed.

THEOREM 3. Let $(X, T)$ be a transformation group. Then:

(1) $L=\{z \mid z \in X \times X \cdot \overline{z T} \subset P\}=\bigcup\{\overline{z T} \mid z \in X \times X \cdot \overline{z T} \subset P\}$.

(2) The following statements are equivalent: (i) $P=L$; (ii) If $z \in P$, then $\overline{z T} \subset P$.

(3) If $P$ is closed in $X \times X$, then $P=L$ and $P$ is an invariant closed equivalence relation in $X$.

Proof. Use Lemmas 4 and 5 .

REMARK 4. Simple examples can be constructed in which $P=L$ and yet $P$ is not closed in $X \times X$.

REMARK 5. The following statements hold:

(1) $P$ closed in $X \times X$ implies $P=L$; simple examples show that the converse fails.

(2) $P=L$ implies $P(X \times X)=P(X) \times P(X)$; the status of the converse is not known.

(3) $P(X \times X)=P(X) \times P(X)$ implies $P$ is transitive; the status of the converse is not known.

REMARK 6. Let $(X, T)$ and $(Y, T)$ be transformation groups. Then:

(1) $L(X) \times L(Y)=L(X \times Y)$;

(2) $M(X) \times M(Y)=M(X \times Y)$;

(3) $P(X) \times L(Y) \subset P(X \times Y)$;

(4) $L(X) \times P(Y) \subset P(X \times Y)$;

(5) $Q(X) \times M(Y) \subset Q(X \times Y)$;

(6) $M(X) \times Q(Y) \subset Q(X \times Y)$;

the proof uses the method of Theorem 2 . 
The next sequence of theorems and remarks investigates the productivity of the relations $L, M$, and $P$. In the case of $P$, productivity is established only under limited hypotheses.

REMARK 7. Let $\left(\left(X_{i}, T\right) \mid i \in I\right)$ be a family of transformation groups with a common phase group $T$ and whose phase spaces are uniform spaces which are not necessarily compact. For $j \in I$, let $\theta_{j}:\left(X_{i \in I} X_{i}\right)^{2} \rightarrow X_{j}^{2}$ be the map such that $\left(\left(x_{i} \mid i \in I\right),\left(y_{i} \mid i \in I\right)\right) \theta_{j}=\left(x_{j}, y_{j}\right)$ and let $\theta:\left(X_{i \in I} X_{i}\right)^{2} \rightarrow X_{i \in I} X_{i}^{2}$ be the map such that $\left(\left(x_{i} \mid i \in I\right),\left(y_{i} \mid i \in I\right)\right) \theta=\left(\left(x_{i}, y_{i}\right) \mid i \in I\right)$ for $\left(\left(x_{i} \mid i \in I\right),\left(y_{i} \mid i \in I\right)\right)$ $\in\left(X_{i \in I} X_{i}\right)^{2}$. Then:

(1) $z \in\left(X_{i \in I} X_{i}\right)^{2}$ implies $z \theta=\left(z \theta_{i} \mid i \in I\right)$.

(2) If $j \in I$, then $\theta_{j}$ is an open homomorphism, called the canonical homomorphism, of $\left(\left(X_{i \in I} X_{i}\right)^{2}, T\right)$ onto $\left(X_{j}^{2}, T\right)$.

(3) $\theta$ is an isomorphism, called the canonical isomorphism, of $\left.\left(\chi_{i \in I} X_{i}\right)^{2}, T\right)$ onto $\left(X_{i \in I} X_{i}^{2}, T\right)$. When no ambiguity occurs the symbol for $\theta$ may be omitted from a statement.

(4) If $j \in I$, then $\theta_{j}$ maps $R\left(X_{i \in I} X_{i}, T\right)$ onto $R\left(X_{j}, T\right)$, where $R$ is $P$ or $Q$ or $L$ or $M$.

(5) 0 maps $R\left(\chi_{i \in I} X_{i}, T\right)$ into $\chi_{i \in I} R\left(X_{i}, T\right)$, where $R$ is $P$ or $Q$ or $L$ or $M$.

(6) According to (4) and (5), we may say that $R\left(X_{i \in I} X_{i}, T\right)$ is a canonical subdirect product of $\left(R\left(X_{i}, T\right) \mid i \in I\right)$, where $R$ is $P$ or $Q$ or $L$ or $M$.

LEMma 6. Let $n$ be a positive integer, let $\left(X_{1}, T\right), \cdots,\left(X_{n}, T\right)$ be transformation groups, let $\left(x_{i}, y_{i}\right) \in L\left(X_{i}, T\right)$ and let $\alpha_{i}$ be an index of $X_{i}(i=1, \cdots, n)$. Then there exists $A \in \mathscr{A}$ such that $\left(x_{i}, y_{i}\right) A \subset \alpha_{i}$ for each $i=1, \cdots, n$.

Proof. There exists $A_{1} \in \mathscr{A}$ such that $\left(x_{1}, y_{1}\right) A_{1} \subset \alpha_{1}$. Let $K_{1} \in \mathscr{K}$ such that $T=A_{1} K_{1}$. There exists $A_{2} \in \mathscr{A}$ such that $\left(x_{2}, y_{2}\right) A_{2} K_{1}^{-1} \subset \alpha_{2}$. Let $K_{2} \in \mathscr{K}$ for which $T=A_{2} K_{2}$. There exists $A_{3} \in \mathscr{A}$ such that $\left(x_{3}, y_{3}\right) A_{3}\left(K_{1} K_{2}\right)^{-1} \subset \alpha_{3}$. Let $K_{3} \in \mathscr{K}$ for which $T=A_{3} K_{3}$. This process is continued. Hence, there exist subsets $A_{1}, \cdots, A_{n}, K_{1}, \cdots, K_{n}$ of $T$ such that $T=A_{i} K_{i}, K_{i} \in \mathscr{K}$, and $\left(x_{i}, y_{i}\right) A_{i}\left(\prod_{j=1}^{i-1} K_{j}\right)^{-1} \subset \alpha_{i}$ for each $i=1, \cdots, n\left(K_{0}=\right.$ identity element in $\left.T\right)$. Define $A=\bigcap_{i=1}^{n} A_{i}\left(\prod_{j=1}^{i-1} K_{j}\right)^{-1}$. Now $\left(x_{i}, y_{i}\right) A \subset \alpha_{i}$ for $i=1, \cdots, n$. By Lemma $3, A \in \mathscr{A}$. The proof is completed.

Lemma 7. Let $n$ be a positive integer, let $\left(X_{1}, T\right), \cdots,\left(X_{n}, T\right)$ be transformation groups. For $i=1, \cdots, n$, let $\left(x_{i}, y_{i}\right) \in M\left(X_{i}, T\right)$, let $\alpha_{i}$ be an index of $X_{i}$, and let $U_{i} \in \mathscr{N}_{x_{i}}, V_{i} \in \mathscr{N}_{y_{i}}$. Then there exists $p_{i} \in U_{i}, q_{i} \in V_{i}(i=1, \cdots, n)$ and $A \in \mathscr{A}$ such that $\left(p_{i}, q_{i}\right) A \subset \alpha_{i}$ for $i=1, \cdots, n$.

Proof. There exists $p_{1} \in U_{1}, q_{1} \in V_{1}$, and $A_{1} \in \mathscr{A}$ such that $\left(p_{1}, q_{1}\right) A_{1} \subset \alpha_{1}$. Let $K_{1} \in \mathscr{K}$ for which $T=A_{1} K_{1}$. There exists $p_{2} \in U_{2}, q_{2} \in V_{2}$, and $A_{2} \in \mathscr{A}$ such that $\left(p_{2}, q_{2}\right) A_{2} K_{1}^{-1} \subset \alpha_{2}$. Let $K_{2} \in \mathscr{K}$ for which $T=A_{2} K_{2}$. There exists $p_{3} \in U_{3}$, $q_{3} \in V_{3}$, and $A_{3} \in \mathscr{A}$ such that $\left(p_{3}, q_{3}\right) A_{3}\left(K_{1} K_{2}\right)^{-1} \subset \alpha_{3}$. Let $K_{3} \in \mathscr{K}$ for which 
$T=A_{3} K_{3}$. This process is continued. Hence, there exists $p_{i} \in U_{i}, q_{i} \in V_{i}(i=1, \cdots, n$ and subsets $A_{1}, \cdots, A_{n}, K_{1}, \cdots, K_{n}$ of $T$ such that $T=A_{i} K_{i}, K_{i} \in \mathscr{K}$, and $\left(p_{i}, q_{i}\right) A_{i}\left(\prod_{j=1}^{i-1} K_{j}\right)^{-1} \subset \alpha_{i}$ for each $i=1, \cdots, n\left(K_{0}=\right.$ identity element in $\left.T\right)$. Define $A=\bigcap_{i=1}^{n} A_{i}\left(\prod_{j=1}^{i-1} K_{j}\right)^{-1}$. Now $\left(p_{i}, q_{i}\right) A \subset \alpha_{i}$ for $i=1, \cdots, n$. By Lemma 3 , $A \in \mathscr{A}$. The proof is completed.

THEOREM 4. Let $\left(\left(X_{i}, T\right) \mid i \in I\right)$ be a family of transformation groups, and let $\theta$ be the canonical isomorphism of $\left(\left(X_{i \in I} X_{i}\right)^{2}, T\right)$ onto $\left(X_{i \in I} X_{i}^{2}, T\right)$. Then

(1) $L\left(X_{i \in I} X_{i}, T\right) \theta=X_{i \in I} L\left(X_{i}, T\right)$.

(2) $M\left(X_{i \in I} X_{i}, T\right) \theta=\chi_{i \in I} M\left(X_{i}, T\right)$.

Proof. (1) By Remark 7, $L\left(X_{i \in I} X_{i}, T\right) \theta \subset \chi_{i \in I} L\left(X_{i}, T\right)$. It remains to show $L\left(\chi_{i \in I} X_{i}, T\right) \theta \supset \chi_{i \in I} L\left(X_{i}, T\right)$. Let $\left(\left(x_{i}, y_{i}\right) \mid i \in I\right) \in \chi_{i \in I} L\left(X_{i}, T\right)$ whence $\left(x_{i}, y_{i}\right) \in L\left(X_{i}, T\right)$ for each $i \in I$. It is enough to show $\left(\left(x_{i} \mid i \in I\right),\left(y_{i} \mid i \in I\right)\right)$ $\in L\left(X_{i \in I} X_{i}, T\right)$. Let $J$ be a finite nonvacuous subset of $I$, and for each $j \in J$ let $\alpha_{j}$ be an index of $X_{j}$. It is sufficient to prove that there exists a syndetic subset $A$ of $T$ such that $\left(x_{j}, y_{j}\right) A \subset \alpha_{j}$ for each $j \in J$. But Lemma 6 states exactly this. The proof of (1) is completed.

(2) By Remark 7, $M\left(\chi_{\iota \in I} X_{i}, T\right) \theta \subset \chi_{i \in I} M\left(X_{i}, T\right)$. It remains to show $M\left(\chi_{i \in I} X_{i}, T\right) \supset \chi_{i \in I} M\left(X_{i}, T\right)$. Let $\left(\left(x_{i}, y_{i}\right) \mid i \in I\right) \in \chi_{i \in I} M\left(X_{i}, T\right)$ whence $\left(x_{i}, y_{i}\right) \in M\left(X_{i}, T\right)$ for each $i \in I$. It is enough to show $\left(\left(x_{i} \mid i \in I\right),\left(y_{i} \mid i \in I\right)\right)$ $\in M\left(\chi_{i \in I} X_{i}, T\right)$. Let $J$ be a finite nonvacuous subset of $I$. For each $j \in J$ let $\alpha_{j}$ be an index of $X_{j}$, and for each $j \in J$, let $U_{j}, V_{j}$ be neighborhoods of $x_{j}, y_{j}$ in $X_{j}$. It is sufficient to prove that there exist $p_{j} \in U_{j}, q_{j} \in V_{j}(j \in J)$ and a syndetic subset $A$ of $T$ such that $\left(p_{j}, q_{j}\right) A \subset \alpha_{j}$ for each $j \in J$. But Lemma 7 states exactly this. The proof is completed.

REMARK 8. Let $\left(\left(X_{i}, T\right) \mid i \in I\right)$ be a family of transformation groups, and let $P\left(X_{i}, T\right)$ be closed in $X_{i}^{2}$ for each $i \in I$. Then $P\left(X_{i \in I} X_{i}, T\right) \theta=\chi_{i \in I} P\left(X_{i}, T\right)$. Use Theorem 4 and Remark 7.

THEOREM 5. Let $\left(\left(X_{i}, T\right) \mid i \in I\right)$ be a family of transformation groups. Then the following statements are equivalent:

(1) $P\left(X_{i \in I} X_{i}, T\right)$ is closed in $\left(X_{i \in I} X_{i}\right)^{2}$.

(2) For each $i \in I, P\left(X_{i}, T\right)$ is closed in $X_{i}^{2}$.

Proof. Use Remark 7 and Remark 8.

THEOREM 6. Let $\left(\left(X_{i}, T\right) \mid i \in I\right)$ be a family of transformation groups. Then:

(1) $\left(X_{i \in I} X_{i}, T\right)$ is [repletely distal] [regionally repletely distal] if and only if $\left(X_{i}, T\right)$ is [repletely distal] [regionally repletely distal] for each $i \in I$.

(2) There exists a least invariant closed equivalence relation $S$ in $X$ such that $(X / S, T)$ is [repletely distal] [regionally repletely distal].

Proof. Use Theorem 4 and [5, Theorem 1 and Remark 8].

This final section describes various relationships between $L, M, P$, and $Q$ when various types of hypotheses are imposed upon the transformation group $(X, T)$. 
Definition 4. Let $(X, T)$ be a transformation group where $X$ is not necessarily compact. Then $(X, T)$ is said to be coterminous provided that $P=Q=L=M$.

THEOREM 7. Let $(X, T)$ be a transformation group. Then the following statements are equivalent:

(1) $(X, T)$ is coterminous.

(2) $P \supset Q$.

Proof. Use Theorem 3 and Remark 1.

THEOREM 8. Let $(X, T)$ be a tranformation group. Let $S($ distal) be the distal structure relation of $(X, T)$ (see [5, Definition 4]). The following statements are pairwise equivalent:

(1) $P=S($ distal $)$.

(2) $P=L=S($ distal $)$.

(3) $P$ is closed in $X \times X$.

Proof. Use Theorem 3 and [5, Theorem 2].

THEOREM 9. Let $(X, T)$ be a transformation group where $X$ is not necessarily compact. Let $T$ be abelian, let some point of $X$ be fixed under $(X, T)$, and let $x \in X$ such that $\overline{x T}=X$. Then:

(1) $x T \times x T \subset P$.

(2) $\bar{P}=Q=X \times X$.

Proof. It is sufficient to prove (1). Let $t, s \in T$, and let $\alpha \in \mathscr{U}$. Let $y \in X$ such that $y T=y$. Choose $U \in \mathscr{N}_{y}$ so that $U \times U \subset \alpha$. Since $y t^{-1} s=y$, there exists $V \in \mathscr{N}_{y}$ such that $V \subset U$ and $V t^{-1} s \subset U$. There exists $r \in T$ such that $x t r \in V$ whence $x t r \in U, x s r=x t r t^{-1} s \in V t^{-1} s \subset U$, and $(x t r, x s r) \in U \times U \subset \alpha$. The proof is completed.

TheOrem 10. Let $(X, T)$ be a distal transformation group. Then $Q=M$.

Proof. Let $(x, y) \in Q$. It is enough to show $(x, y) \in M$. Let $\alpha \in \mathscr{U}$, and let $W \in \mathscr{N}_{(x, y)}$. Choose $U \in \mathscr{N}_{x}$, and $V \in \mathscr{N}_{y}$ such that $U \times V \subset W$. Choose $\gamma \in \mathscr{U}$ such that $\gamma^{4} \subset \alpha$. There exist $\left(x_{1}, y_{1}\right) \in U \times V$ and $t \in T$ such that $\left(x_{1} t, y_{1} t\right) \in \gamma$, whence $y_{1} t \in x_{1} t \gamma$. Since $(X, T)$ is distal, $\left(X^{2}, T\right)$ is pointwise almost periodic [4, Theorem 1]. Hence, there exists $B \in \mathscr{A}$ such that $\left(x_{1} t, y_{1} t\right) B \subset x_{1} t \gamma \times y_{1} t \gamma$. Now $\left(x_{1} t, y_{1} t\right) B \subset \gamma^{4} \subset \alpha$. Define $A=t B$, whence $\left(x_{1}, y_{1}\right) \in U \times V \subset W$ and $\left(x_{1}, y_{1}\right) A \subset \alpha$. The proof is completed.

LEMMA 8. Let $(X, T)$ be a transformation group where $X$ is not necessarily compact. Let $T$ be abelian, let $x \in X$, let $t, s \in T$, and let $(X, T)$ be recursive at $x$. Then $(X \times X, T)$ is recursive at $(x t, x s)$.

Proof. Let $W \in \mathscr{N}_{(x t, x s)}$. Choose $U \in \mathscr{N}_{x t}$ and $V \in \mathscr{N}_{x s}$ such that $U \times V \subset W$. There exist $U_{1}, V_{1} \in \mathscr{N}_{x}$ such that $U_{1} t \subset U$ and $V_{1} s \subset V$. There exists an admissible subset $A$ of $T$ such that $x A \subset U_{1} \cap V_{1}$. Then $x t A \subset U, x s A \subset V$, whence $(x t, x s) A \subset W$. The proof is completed. 
THEOREM 11. Let $(X, T)$ be a minimal transformation group where $T$ is abelian. Then $Q=M$.

Proof. Let $z \in Q$. We show $z \in M$. Let $\alpha$ be an open index of $X$ and let $U$ be an open neighborhood of $z$. There exists $w \in U$ and $t \in T$ such that $w t \in \alpha$. Choose $V \in \mathscr{N}_{w}$ such that $V \subset U$ and $V t \subset \alpha$. There exists $x \in X$ and $s \in T$ such that $z_{1}=(x, x s) \in V$. Since $z_{1}$ is almost periodic under $(X \times X, T)$ by Lemma 8 , and $\alpha \cap z_{1} T \neq \varnothing$, there exists $A \in \mathscr{A}$ such that $z_{1} A \subset \alpha$. Hence $z \in M$. The proof is completed.

Lemma 9. Let $(X, T)$ be a transformation group, let $x \in X$, let $T$ be almost periodic at $x$, let $U \in \mathscr{N}_{x}$, and let $\overline{x T}$ be infinite. Then there exists $t \in T$ such that $x t \in U$ and $x t \neq x$.

Proof. There exists $A \in \mathscr{A}$ and a finite subset $K$ of $T$ such that $T=A K$ and $x A \subset U$. It is enough to show $x A \neq x$. Suppose $x A=x$. Then $x T=x A K=x K$ and $\overline{x T}=x K$ which is a contradiction. The proof is completed.

THEOREM 12. Let $(X, T)$ be an expansive pointwise almost periodic transformation group. Suppose for each $x \in X, \overline{x T}$ is infinite and let $T$ be abelian. Then

$$
M \neq \Delta .
$$

Proof. There exists an open index $\delta$ of $X$ such that $z \in X \times X \cap \Delta^{\prime}$ implies $z T \cap \delta^{\prime} \neq \varnothing$. For $\alpha \in \mathscr{U}$, let $E_{\alpha}$ be the set of all $z \in X \times X$ such that $z$ is almost periodic under $\left(X^{2}, T\right)$ and $z T \cap \alpha \neq \emptyset$. By Lemma $9,\left\{\bar{E}_{\alpha} \cap \delta^{\prime} \mid \alpha \in \mathscr{U}\right\}$ is a closed filter base on $X \times X$. It follows that $\delta^{\prime} \cap \bigcap_{\alpha \in \mathscr{U}} \bar{E}_{\alpha} \neq \varnothing$. Since $M \supset \bigcap_{\alpha \in \mathscr{U}} \bar{E}_{\alpha}$, the proof is completed.

THEOREM 13. Let $(X, T)$ be a regionally mixing transformation group for which $T$ is not compact. Then:

(1) $Q=X \times X$.

(2) If $T$ is abelian and $(X, T)$ is minimal, then $P \neq Q$.

Proof. (1) Let $(x, y) \in X \times X$. It is sufficient to show $(x, y) \in Q$. Let $\alpha \in \mathscr{I}$, $U \in \mathscr{N}_{x}, V \in \mathscr{N}_{y}$. It is sufficient to show $(U \times V) T \cap \alpha \neq \emptyset$. We may assume $V \times V \subset \alpha$. There exists $K \in \mathscr{K}$ such that $t \in T-K$ implies $U t \cap V \neq \varnothing$. There exists $H \in \mathscr{K}$ for which $V t \cap V \neq \varnothing$ for $t \in T-H$. Now $((T-H) \cap(T-K))^{\prime}=H \cup K$ and hence $(T-K) \cap(T-H) \neq \varnothing$ since $T \notin \mathscr{K}$ and $H \cup K \in \mathscr{K}$. Choose $s \in(T-K) \cap(T-H), x_{1} \in U$, and $y_{1} \in V$ such that $x_{1} s \in V$ and $y_{1} s \in V$. Now $\left(x_{1}, y_{1}\right) \in U \times V$ and $\left(x_{1} s, y_{1} s\right) \in \alpha$, whence $(U \times V) T \cap \alpha \neq \varnothing$. Therefore $(x, y) \in Q$.

(2) By $[8,10.07]$ if $x \neq y$ and $y \in x T$, then $(x, y) \notin P$. Therefore $P \neq X \times X=Q$. The proof is completed.

Lemma 10. Let $T$ be a topological group and let $R$ be a right replete subset of $T$, that is to say, for each $K \in \mathscr{K}$, there exists $t \in T$ such that $K t \subset R$. Then: 
(1) $t R \cap R \neq \varnothing$ for all $t \in T$.

(2) $T=A R$ for any $A \in \mathscr{A}$.

Proof. (1) Let $t \in T$. Let $K=\left\{e, t^{-1}\right\}$. Since there exists $s \in T$ such that $K s \subset R, s \in t R \cap R$.

(2) Let $A \in \mathscr{A}$ and $K \in \mathscr{K}$ such that $T=A K$. Since there exists $s \in T$ such that $K s \subset R, T=A K s \subset A R$ and $T=A R$. The proof is completed.

THEOREM 14. The following statements are equivalent:

(1) $T$ is uniformly equicontinuous.

(2) For each $\alpha \in \mathscr{U}$, there exist a right replete subset $R$ of $T$ and $\beta \in \mathscr{U}$ such that $(x, y) \in \beta$ implies $(x, y) R \subset \alpha$.

Proof. It is sufficient to show (2) implies (1). We first show $Q=P$. It is sufficient to show $Q \subset P$. Let $(x, y) \in Q$. Let $\alpha \in \mathscr{U}$, let $R$ be a replete subset of $T$, and $\beta, \gamma \in \mathscr{U}$ such that $\beta^{3} \subset \alpha$ and $\gamma R \subset \beta$. There exist $x_{1} \in x \gamma, y_{1} \in y \gamma$ and $t \in T$ such that $\left(x_{1} t, y_{1} t\right) \in \gamma$. Now, by Lemma 10 , we may choose $s \in t R \cap R$, whence $(x s, y s)=\left(x s, x_{1} s\right)\left(x_{1} s, y_{1} s\right)\left(y_{1} s, y s\right) \in \beta^{3} \subset \alpha$. Therefore $(x, y) \in P$ and $P \subset Q$. By Theorem 7, $P=L=M=Q$. It remains to show that $L \subset \Delta$. Let $(x, y) \in L$. Let $\alpha \in \mathscr{U}$, and let $R$ and $\beta$ be such that $\beta R \subset \alpha$. There exists $A \in \mathscr{A}$ such that $(x, y) A \subset \beta$, whence $(x, y) A R \subset \beta R \subset \alpha$. But by Lemma 10, $T=A R$ whence $(x, y) T \subset \alpha$ for each $\alpha \in \mathscr{U}$. Therefore $x=y$ and $L=Q=\Delta$. The proof is completed.

REMARK 9. Theorem 14 weakens the hypothesis of a theorem by John D. Baum [2] in that he required $T$ to be abelian and $R$ to be a replete semigroup of $T$.

\title{
BIBLIOGRAPHY
}

1. Joseph Auslander, Mean-L-stable systems, Illinois J. Math. 3 (1959), 566-579.

2. John D. Baum, An equicontinuity condition for transformation groups, Proc. Amer. Math. Soc. 12 (1961), 30-32.

3. N. Bourbaki, Elements de mathematique. I. Les structures fondamentales de l'analyse, Livre III, Topologie générale, Chapitre I, Actualités Sci. Ind. No. 858, Hermann, Paris, 1940.

4. Robert Ellis, Distal transformation groups, Pacific J. Math. 8 (1958), 401-405.

5. Robert Ellis and W. H. Gottschalk, Homomorphisms of transformation groups, Trans. Amer. Math. Soc. 94 (1960), 258-271.

6. E. E. Floyd, A nonhomogeneous minimal set, Bull. Amer. Math. Soc. 55 (1949), 957-960.

7. W. H. Gottschalk, Minimal sets: An introduction to topological dynamics, Bull. Amer. Math. Soc. 64 (1958), 336-351.

8. W. H. Gottschalk and G. A. Hedlund, Topological dynamics, Amer. Math. Soc. Colloq. Publ. Vol. 36, Amer. Math. Soc., Providence, R. I., 1955.

9. G. A. Hedlund, Sturmian minimal sets, Amer. J. Math. 66 (1944), 605-620.

10. J. L. Kelley, General topology, Van Nostrand, New York, 1955.

\author{
University of Pennsylvania, \\ Philadel phita, Pennsylvania \\ UnIVAC Division, \\ SPERRY RAND CORPORATION \\ Philadelphia, Pennsylvania
}

